\title{
All Optical Bidirectional Wavelength Conversion Using Single Wide Band Traveling Wave Semiconductor Optical Amplifiers
}

\author{
Abd El-naser A. \\ Mohamed \\ Electronics and Electrical \\ Communications Engineering \\ Department, Faculty of Electronic \\ Engineering, Menoufia University. \\ Egypt.
}

\author{
Ahmed Rashed \\ Electronics and Electrical \\ Communications Engineering \\ Department, Faculty of Electronic \\ Engineering, Menoufia University. \\ Egypt.
}

\author{
Mohamed Basuony Aboelazm \\ Benha for electronic industry \\ ministry of defense and military \\ production, Egypt.
}

\author{
Mohamed F. Tabbour \\ Electronics and Electrical \\ Communications Engineering \\ Department, Faculty of Electronic \\ Engineering, Menoufia University. \\ Egypt.
}

\begin{abstract}
Wavelength division multiplexing (WDM) is a very important technique to utilize the bandwidth of optical fiber. At different network nodes, it's requires to add or drop wavelength. The wavelength converter, responsible for converting the wavelength of signal, to another wavelength up or down from the original value. This paper presents a proposed model to generate inverted and non-inverted wavelength conversion by using single wide band traveling wave semiconductor optical amplifier (WBSOA), based on cross gain modulation (XGM). The investigation of conversion efficiency $(\eta)$ and quality factor $(Q)$, versus pump power ranging from $-30 \mathrm{dBm}$ to $0 \mathrm{dBm}$, and input signal power of $0 \mathrm{dBm}$ with data rate $25 \mathrm{~Gb} / \mathrm{s}$, studies for up and down wavelength conversion "copropagation" and "counter-propagation" respectively, along c-band. All simulations in this study are performed by optisystem ver. 7 .
\end{abstract}

Keywords: WDM, All optical wavelength conversion, (WBSOA), optical to electrical to optical $(\mathrm{O} / \mathrm{E} / \mathrm{O})$, conversion efficiency, quality factor.

\section{Introduction}

WDM multiplexes a number of optical signals onto a single optical fiber by using different wavelengths, The capacity of WDM based optical communication networks is usually limited by the number of channels available and wavelength congestion. The construction in Fig.1 (a) is apart from network, only two wavelengths have been established in this network, the first wavelength between Node 1 and Node 2 is $\lambda_{1}$, and the second wavelength available between node 2 and node 3 is $\lambda_{2}$. Instant need to set up a light path from node 1 to node 3 directly, under previous condition the construction didn't have more than two wavelengths $\lambda_{1}$ and $\lambda_{2}$. It is impossible to establish a light path because the wavelengths available in two links are different, the network will be blocked. by using a wavelength converter, the data will sent from Node 1 to Node 2 on two different wavelengths $\lambda_{1}$ and $\lambda_{2}$, as in Fig. 1(b). Then at Node 2 we will create a wavelength converting, the data wavelength $\lambda_{2}$, should be change from wavelength $\lambda_{2}$ to $\lambda_{1}$. It is obviously clear, wavelength converter avoided, blocking and adds flexibility in optical network $[1,2]$.

The wavelength conversion process, can take two mechanisms. The $\mathrm{O} / \mathrm{E} / \mathrm{O}$, where the signal originally in the optical domain, and converted to electrical domain, in electrical domain create all signal processing, then converted into the optical domain again. This mechanism has some limitation, such as system complexity, costly and power consumption. The other mechanism is all optical wavelength conversion, all signal processing done in the optical domain without need to enter the electrical domain. The advantage of this technique is simplest, fastest, and power efficient. The reason to choose SOA as a nonlinear device to create wavelength conversion is the dual functionality, the dual functionality of SOA is an enhanced nonlinear device and is also amplifying the optical signal.

The results from this configuration design are two wavelengths. The first one result from forward propagation "co-propagation", and the other signal result from backward propagation "counter-propagation". The Interaction between more than two signals in SOA medium take more than three types of phenomenon as indicate in $[3,4]$. The three important phenomenons are Cross Gain Modulation (XGM), Cross Phase Modulation (XPM) and Four Wave Mixing (FWM). Each phenomenon required some conditions to be created [5]. 


\section{Basic Concepts of XGM}

The schematic for XGM wavelength converters is appears in Fig. 2. The important parameter required to create XGM is the power of the input signal must be greater than pump signal power, and SOA biased current must be enough to deplete the carrier concentration in the active region, hence saturate the SOA $[6,7]$. The pumped signal parameters are, $3 \mathrm{dBm}$ optical signal power and wavelength $1560 \mathrm{~nm}$ perform the desired wavelength, the pump signal will enter the SOA through pump coupler. The input data signal called probe signal has a $5 \mathrm{dBm}$ optical power with wavelength $1565 \mathrm{~nm}$, NRZ pesudo random bit sequence will enter the Mach Zehnder Modulator, the XGM WC is designed based on copropagation configuration. AOWCs based XGM has simple configuration, a large dynamic range of the input optical signal power, high conversion efficiency, polarization immunity and wavelength insensitive. [8-10]. As shown in Fig. 2. The two signals pump and probe will enter the SOA through pump coupler co-propagation, during interaction of two signals in SOA block, the pump signal modulated by the input signal.
The result at the output is signal has the same information as input signal with new wavelength equal pumped wavelength.

\section{Basic proposed Model simulation Architecture}

The simulation schematic diagram Fig. 3(a) separately consists of four parts

\section{i. Transmitter section. \\ ii. Pumped power section. \\ iii. The nonlinear medium section presented by "WBSOA". \\ iv. Receiver and visualizes part, for co- propagation and counter-propagation.}

The transmitter consists of, pesedo-random bit sequence with bit rate equal $25 \mathrm{~Gb} / \mathrm{s}$ of order 7 , the bit sequence will modulate the output of non-returned to zero pulse generator. Non return to zero pulse generators are rectangle shape "Exponential", amplitude value is "1". Continuous wave laser diode with wavelength varied from $1530 \mathrm{~nm}$ to $1565 \mathrm{~nm}$ with optical power $0 \mathrm{dBm}$. Polarization control is seated to create more control on output polarization signal.

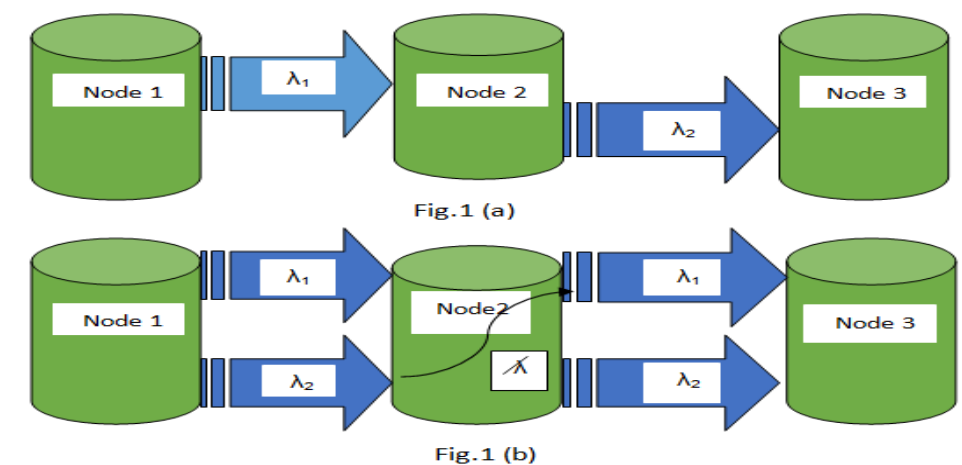

Fig.1 (a) Wavelength-continuity constraint in a wavelength-routed network without converter. (b) Wavelength-continuity constraint in a wavelength-routed network with converter.

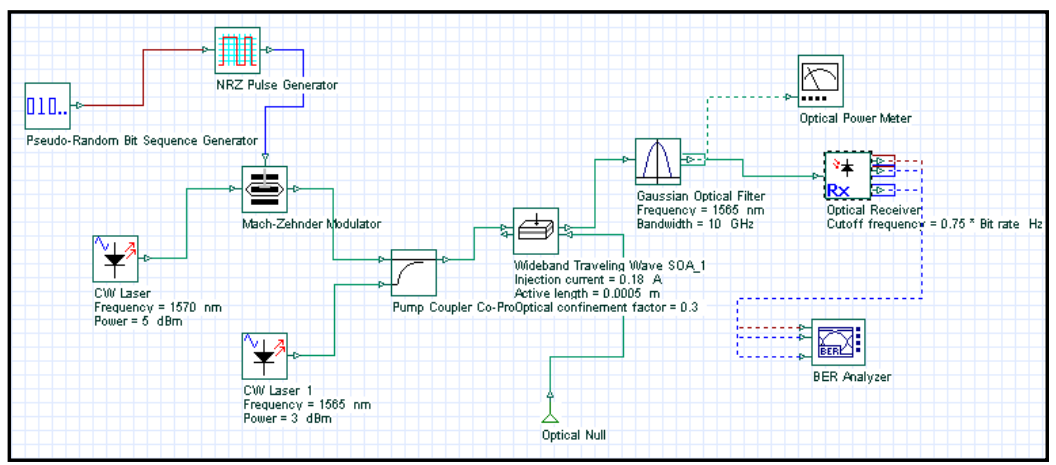

Fig. 2 Simulation schematic for XGM.

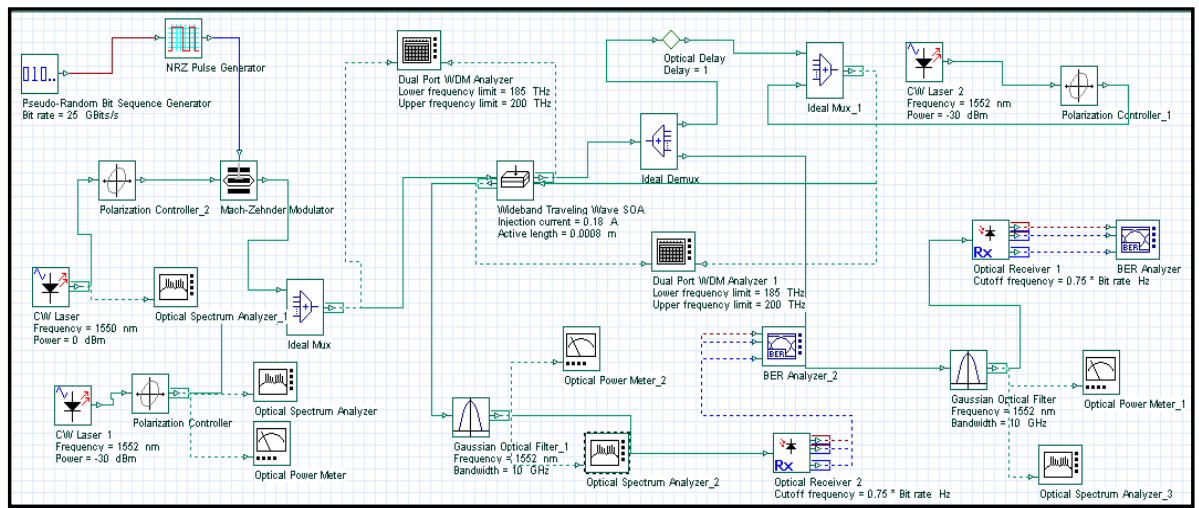

Fig. 3(a) Schematic diagram of circuit simulation. 
The modulator is "Mach Zehander modulator", have extinction ratio $30 \mathrm{~dB}$ mix the non-returned to zero signals and continuous wave laser "CW Laser" signals. Will injecting the pump signal from CW laser_1 \& CW laser 2 for co-propagation and Counter-propagation. The nonlinear medium, is WBSOA, the significant SOA parameter is, the inject current $180 \mathrm{~mA}[5,6]$, the active length $800 \times 10^{-6} \mathrm{~m}$. At the output of WBSOA ideal Demux used to create two signals, each signal carry the same information and wavelength.

The outputs of WBSOA are two, the first one is copropagation output and the second is counter-propagation output, the co-propagation output will enter the receiver part 1, the counter-propagation output will enter the receiver part 2. The curves Fig. 3(b, c) shows the relation between WBSOA gain $(\mathrm{dB})$ versus input power $(\mathrm{dBm})$ of the semiconductor optical amplifier for co-propagation Fig. 3 (b), and counter-propagation Fig. 3(c). In the Fig. 3(b). The amplifier gain is nearly constant from the input signal power $-40 \mathrm{dBm}$ to value below $-25 \mathrm{dBm}$. On the other hand for counter-propagation gain curve the amplifier gain is of low value from input power $-40 \mathrm{dBm}$ to values near $-25 \mathrm{dBm}$.

As the gain of forward direction "co-propagation" decreases the gain of backward "counter-propagation" is increasing, this obviously appears in the area around -20 $\mathrm{dBm}$ to the area around $-10 \mathrm{dBm}$ of input signal power. As will see in later, the area between $-20 \mathrm{dBm}$ to $-10 \mathrm{dBm}$ is the best choice to get maximum value for conversion efficiency, quality factor, and minimum bit error rate, and this area is the best choice to pushing up SOA in nonlinear region.

The main target in this paper is wavelength conversion, so loss in the gain as in Fig. 3(c) not significant in backward direction.

\section{Simulation Results and Discussions}

The start point for this paper comes from previous study of SOA non linearity in "co-propagation" and "counter-propagation" is obtained in [1, 2]. In paper reference [1] the generation of WC is done by using two wavelength shifters, for more amplification the designer uses erbium doped amplifier as a booster amplifier. In paper reference[2] the configuration design changed, the designer used two SOA, and injects signal opposite to each other and take the result in output arm of $2 \times 2$ coupler, the previous design is complex and power inefficiency.

Our design is minimized the circuit by using single WBSOA, as a main part to get the same result as in [1] and [2]. The availability of design can be measured as a function of conversion Efficiency $\eta(\mathrm{dB})=10 \times \log (\mathrm{P}(\lambda$ converted $\left.) / P\left(\mathrm{p}_{\text {umped }}\right)\right)$, and quality factor. The investigation of the proposed design is done for studying the system performance in up and down WC co-propagation and counter-propagation, for varying the pump signal from -30 $\mathrm{dBm}$ to $0 \mathrm{dBm}$ and wavelength varying in $\mathrm{C}$-band, with input bit rate $25 \mathrm{~Gb} / \mathrm{s}$ and input signal power $0 \mathrm{dBm}$.

\subsection{Up Wavelength Conversion Investigation.}

Choose apart from C-band to create the up WC investigation and use configuration as in Fig. 3(a), the incoming signal wavelength is equal to $1550 \mathrm{~nm}$, this signal will be converted up conversion until $1560 \mathrm{~nm}$ in ten steps each step vary $2 \mathrm{~nm}$ from the original signal and investigate at each wavelength shift the conversion efficiency and quality factor, to choice suitable wavelength shift "wavelength detuning" to get maximum performance and availability of the design.

\subsubsection{Up wavelength conversion forward propagation "Co-propagation".}

The conversion efficiency value is not big between each wavelength shifts "detuning", but still at $2 \mathrm{~nm}$ the conversion efficiency is greater than other wave length shift, the area between $-20 \mathrm{dBm}$ to $-10 \mathrm{dBm}$ the conversion efficiency for all wavelength shifts are nearly the same, as shown in Fig. 4(a). The greater value for all wavelength detuning is limited in area of pump power from $-15 \mathrm{dBm}$ to $-5 \mathrm{dBm}$. The greater quality factor value is 4.3 done at pump power $-10 \mathrm{dBm}$ and wavelength shift $4 \mathrm{~nm}$. The minimum BER value is $4.8 \times 10^{-6}$ done at pump power $-10 \mathrm{dBm}$ and wavelength shift $4 \mathrm{~nm}$, as shown in Fig. 4(b).

\subsubsection{Up wavelength conversion backward propagation "Counter-propagation"}

The conversion efficiency at wavelength shift $8 \mathrm{~nm}$ is better than other wavelength shift "detuning", the greater conversion efficiency value is $21.9 \mathrm{~dB}$ done at pump power $-30 \mathrm{dBm}$ and wavelength shift $8 \mathrm{~nm}$. The conversion efficiency at the area from pump power -20 $\mathrm{dBm}$ to $-10 \mathrm{dBm}$ is nearly the same for all wavelength shifts, as shown in Fig. 5(a). The most significant value of the quality factor $(\mathrm{Q})$ still limited around $-10 \mathrm{dBm}$ for all wavelength shifts "detuning". The greater value of quality factor is 4.3 done at pump power $-10 \mathrm{dBm}$ and wavelength shift $2 \mathrm{~nm}$.

\subsection{Down Wavelength Conversion Investigation}

We choose apart in C-band to create the investigation, and use the configuration as in Fig. 3(a). The input wavelength equal to $1555 \mathrm{~nm}$, will be converted down until $1545 \mathrm{~nm}$, in ten steps, each step vary $2 \mathrm{~nm}$ from original signal. At each wavelength shifts, we will investigate the conversion efficiency and quality factor, to choice the perfect and suitable wavelength shift "detuning" to get maximum performance of the proposal design. 


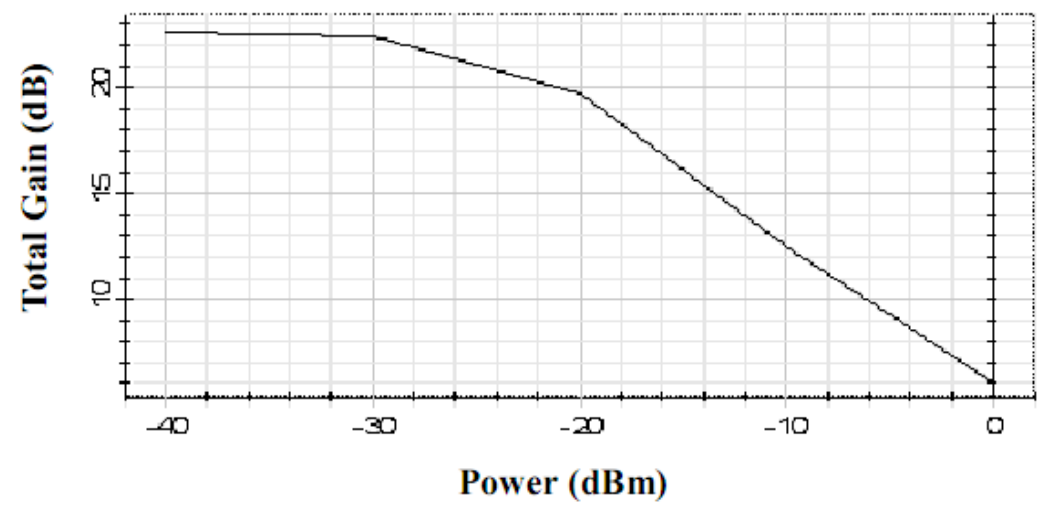

Fig. 3(b) The total gain $(\mathrm{dB})$ variation due to "co-propagation" versus input power $(\mathrm{dBm})$.

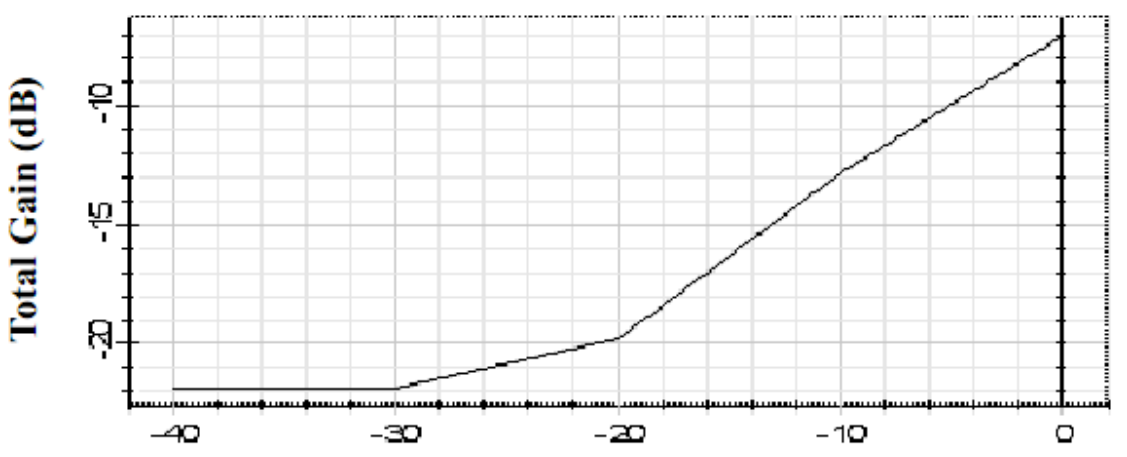

Power (dBm)

Fig. 3(c) The total gain $(\mathrm{dB})$ variation due to "counter-propagation" versus input power $(\mathrm{dBm})$.

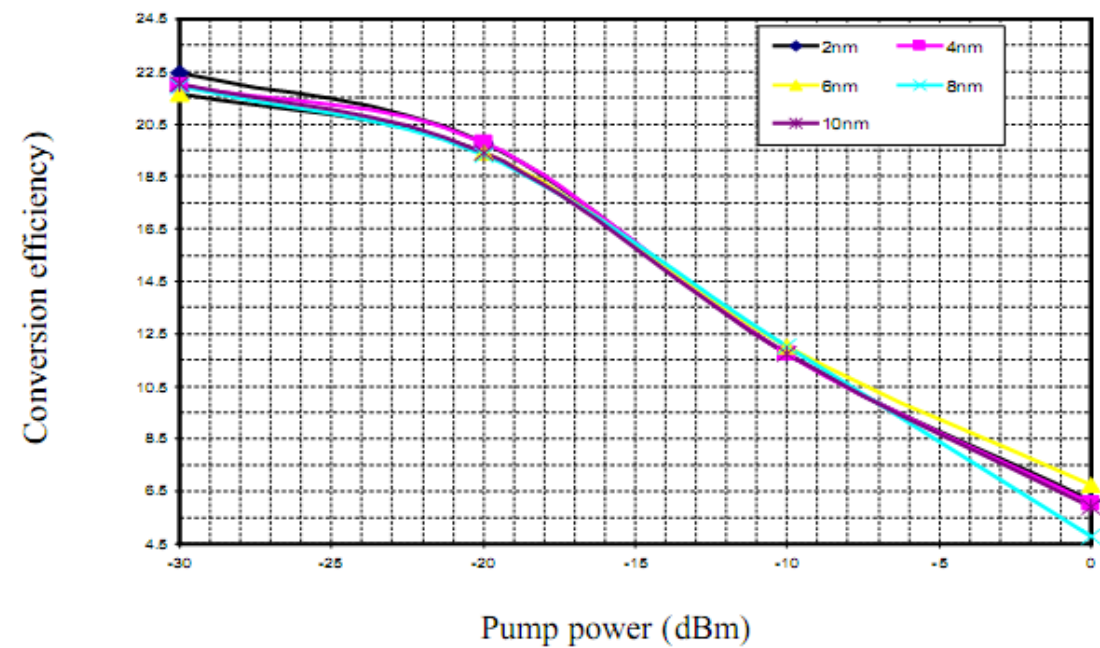

Fig. 4 (a). Conversion efficiency $(\mathrm{dB})$ versus pump power $(\mathrm{dBm})$ for up wavelength conversion "copropagation" at different wavelength shifts.

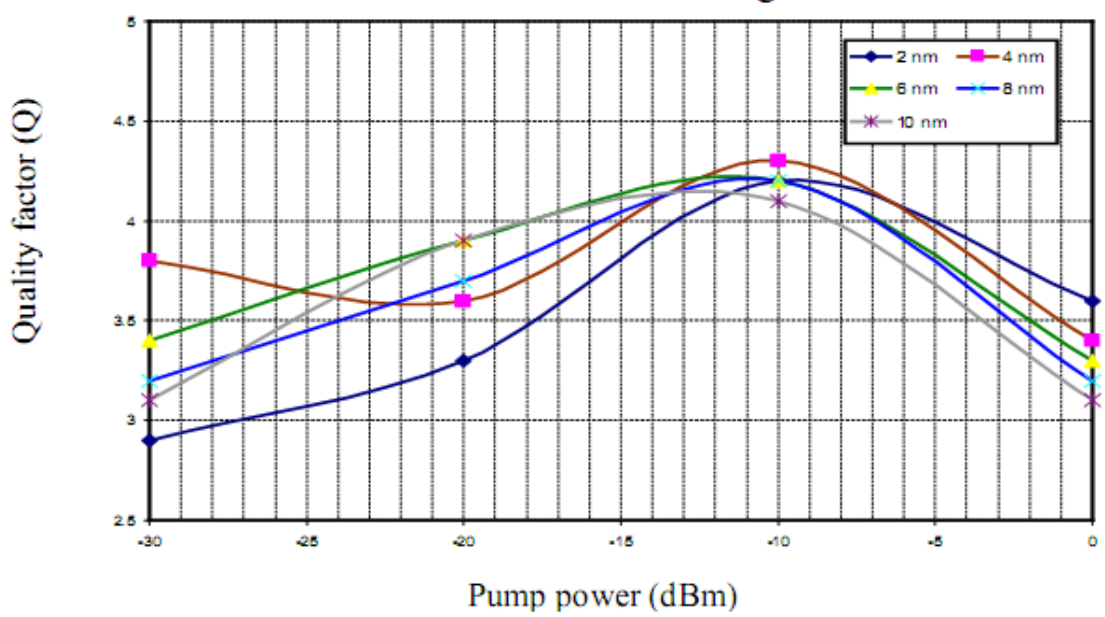

Fig. 4(b) The Quality factor $(\mathrm{Q})$ versus pump power $(\mathrm{dBm})$ for up wavelength conversion "co-propagation" at different wavelength shifts. 


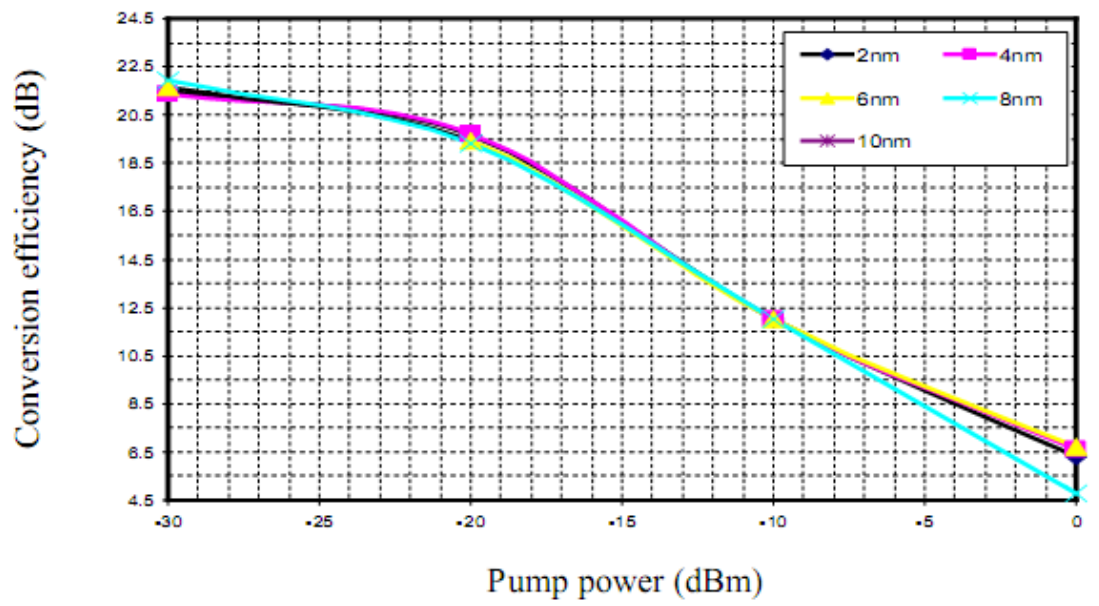

Fig. 5(a) Conversion efficiency $(\mathrm{dB})$ versus pump power $(\mathrm{dBm})$ for up wavelength conversion "counterpropagation" at different wavelength shifts.

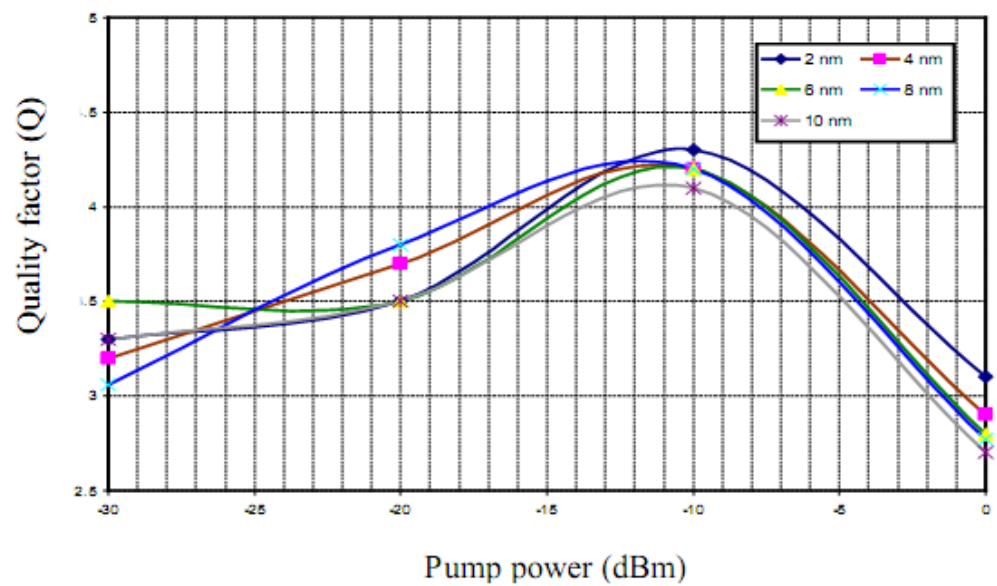

Fig. 5(b) The Quality factor (Q) versus pump power ( $\mathrm{dBm}$ ) for up wavelength conversion "counter-propagation" at different wavelength shifts.

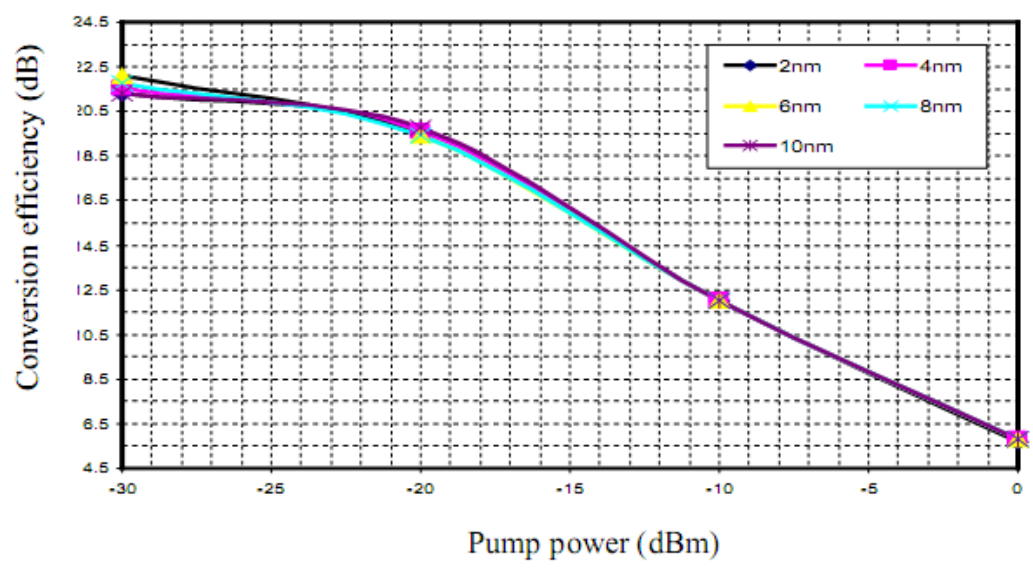

Fig. 6(a) The conversion efficiency $(\mathrm{dB})$ versus pump power $(\mathrm{dBm})$ for down wavelength conversion "copropagation" at different wavelength shifts.

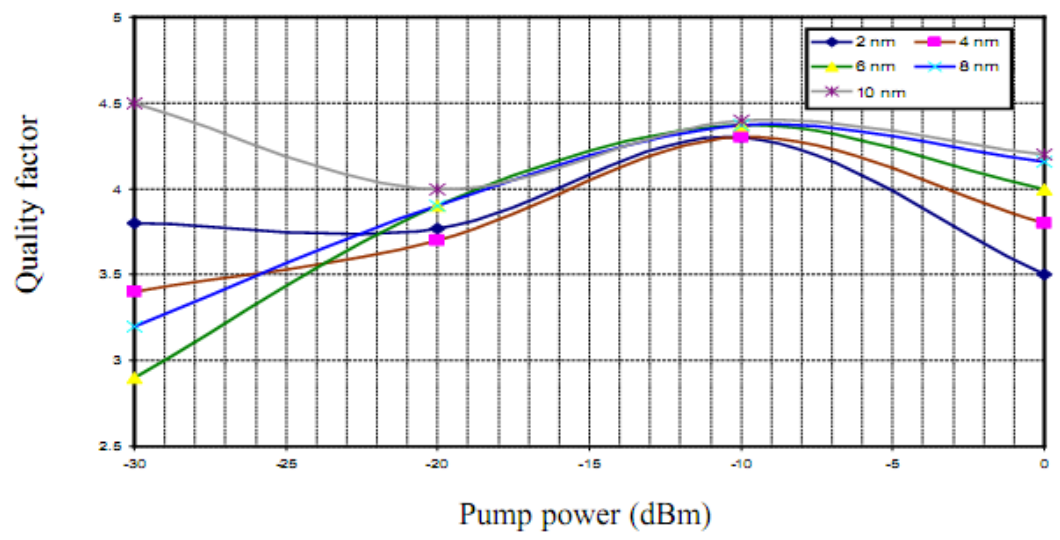

Fig. 6(b). Quality factor $(\mathrm{Q})$ versus pump power $(\mathrm{dBm})$ for down wavelength conversion "co-propagation" at different wavelength shifts. 


\subsubsection{Down wavelength conversion forward propagation "Co-propagation"}

As shown in Fig. 6(a). The greater conversion efficiency value is $22.1 \mathrm{~dB}$, done at pump power $-30 \mathrm{dBm}$ and wavelength shift $6 \mathrm{~nm}$. The lowest conversion efficiency value $5.68 \mathrm{~dB}$ done at pump power $0 \mathrm{dBm}$, and wavelength shift $10 \mathrm{~nm}$, the conversion efficiency result due to pump power from $-20 \mathrm{dBm}$ to $-10 \mathrm{dBm}$ for all wavelength shift nearly the same.

As shown in Fig. 6(b). The greater quality factor value 4.5 done at pumped power $-30 \mathrm{dBm}$ and wavelength shift 10 $\mathrm{nm}$, and the lowest value is 2.9 done at pump power -30 $\mathrm{dBm}$ and wavelength shift $6 \mathrm{~nm}$. At $-10 \mathrm{dBm}$. The minimum BER value is $2.7 \times 10^{-6}$ done at wavelength shift $10 \mathrm{~nm}$ and pump power $-30 \mathrm{dBm}$ and $-10 \mathrm{dBm}$.

\subsection{2 down wavelength conversion backward propagation "Counter-propagation"}

The maximum conversion efficiency is $21.6 \mathrm{~dB}$ done at wavelength shift $6 \mathrm{~nm}$ and pump power $-30 \mathrm{dBm}$, and lowest conversion efficiency value is $3.98 \mathrm{~dB}$, done at wavelength shift $10 \mathrm{~nm}$ and pump power $0 \mathrm{dBm}$.

The graph Fig. 7(b) points to maximum quality factor is done at the pump power value from $-15 \mathrm{dBm}$ to $-5 \mathrm{dBm}$ for all wavelength shifts. The greater quality factor value 4.56 done at pump power $-10 \mathrm{dBm}$ and wavelength shift $10 \mathrm{~nm}$, the lowest quality factor value is 2.9 done at pump power $-30 \mathrm{dBm}$ and wavelength shift $6 \mathrm{~nm}$. The minimum BER value $1.5 \times 10^{-6}$ done at pump power $-10 \mathrm{dBm}$ and wavelength shift $10 \mathrm{~nm}$, as shown in Fig. 7(b).

\section{Special Wavelength Conversion Investigation}

We nominate intermediate value between $-20 \mathrm{dBm}$ and $10 \mathrm{dBm}$, as the best area for pump power, let this value is $-15 \mathrm{dBm}$, the performances of designing will tested at this value. The results collected in Tables $(1,2)$.

Table 1. Performance of up wavelength conversion, where input power and wavelength of $0 \mathrm{dBm}, 1550 \mathrm{~nm}$

Respectively and pump power is $-15 \mathrm{dBm}$, at different wavelength shift "detuning".

\section{Conclusion}

All optical wavelength converters submit a plenty of advantages such as flexibility and simplicity. In this work, wavelength conversion based on XGM, is done by using single wide band traveling wave semiconductor optical amplifier, as performed design has given more advantages to generate inverted and noninverted signal from single WSOA and single source. The performance of the proposed design is tested for up and down wavelength conversion by adapting the variation of wavelength shifted from $2 \mathrm{~nm}$ to $10 \mathrm{~nm}$, pump power from $-30 \mathrm{dBm}$ to $0 \mathrm{dBm}$, with input signal power fixed at $0 \mathrm{dBm}$ with data rate up to $25 \mathrm{~Gb} / \mathrm{s}$. The conversion efficiency and quality factor, versus pump power in different propagation direction "co-propagation \& counter-propagation" are shown at each wavelength shift. It is observed that, as the gain of forward direction decreases the gain of backward direction increases.

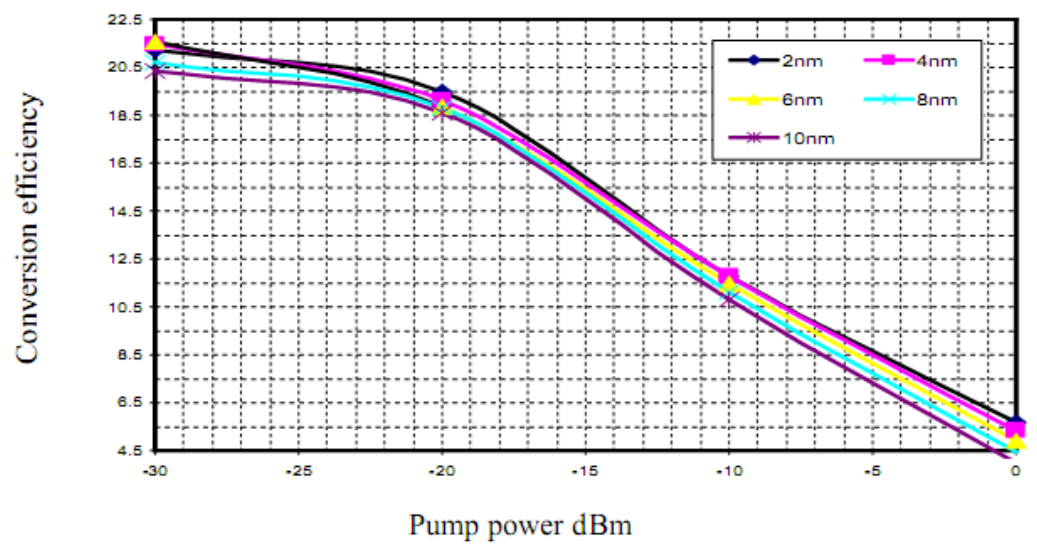

Fig. 7(a) Conversion efficiency $(\mathrm{dB})$ versus pump power $(\mathrm{dBm})$ for down wavelength conversion "counter-propagation" at different wavelength shifts.

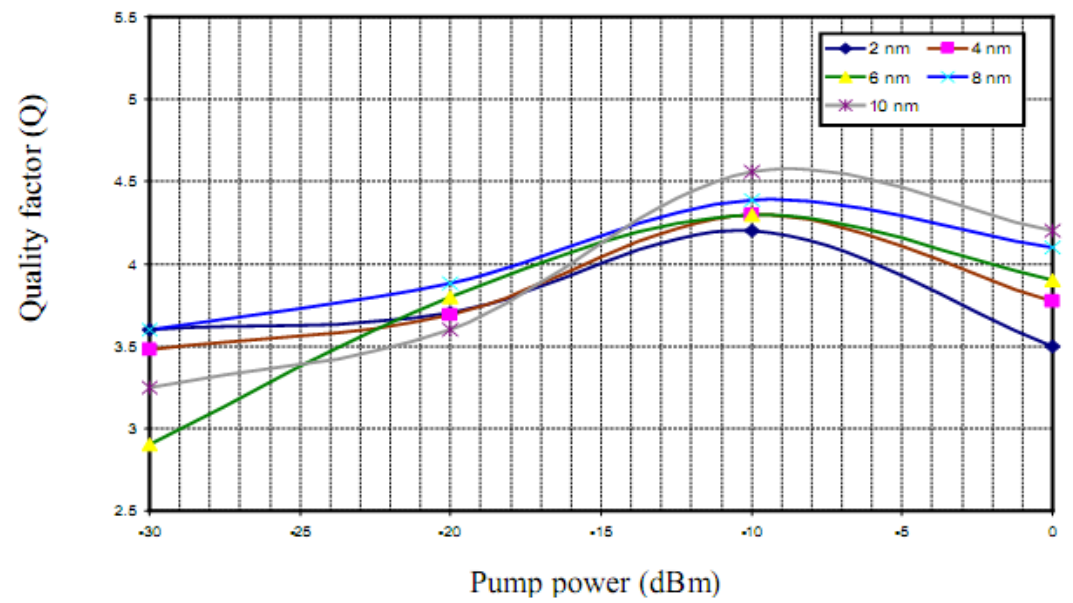

Fig. 7(b). Quality factor $(\mathrm{Q})$ versus pump power $(\mathrm{dBm})$ for down wavelength conversion "counter-propagation" at different wavelength shifts. 
Table 1 Performance of up wavelength conversion, where input power and wavelength is $0 \mathrm{dBm}, 1550 \mathrm{~nm}$, and pump power is -15 $\mathrm{dBm}$, at different wavelength shift "detuning".

\begin{tabular}{|c|c|c|c|c|c|c|c|}
\hline \multirow{3}{*}{$\begin{array}{c}\text { shifted } \\
\text { waveleng } \\
\text { th } \\
\mathrm{nm}\end{array}$} & \multicolumn{7}{|c|}{ Up wavelength conversion } \\
\hline & \multicolumn{3}{|c|}{ Forward "co-propagation" } & \multicolumn{3}{|c|}{$\begin{array}{l}\text { Back ward "counter- } \\
\text { propagation }\end{array}$} & \multirow{2}{*}{$\begin{array}{c}\text { converted } \\
\text { Wavelent } \\
\mathrm{h} \\
\mathrm{nm}\end{array}$} \\
\hline & $\begin{array}{c}\eta \\
(\mathrm{dB})\end{array}$ & $\begin{array}{c}\text { Qualit } \\
y \\
\text { factor }\end{array}$ & BER & $\begin{array}{c}\eta \\
(\mathrm{dB})\end{array}$ & $\begin{array}{c}\text { Qualit } \\
y \\
\text { factor }\end{array}$ & BER & \\
\hline 2 & $\begin{array}{c}16.7 \\
5\end{array}$ & 3.74 & $6 \times 10^{-5}$ & 16.5 & 3.86 & $3.9 \times 10^{-5}$ & 1552 \\
\hline 4 & 16.7 & 3.89 & $3.40 \times 10^{-}$ & $\begin{array}{c}16.7 \\
8\end{array}$ & 3.86 & $3.9 \times 10^{-5}$ & 1554 \\
\hline 6 & $\begin{array}{c}16.7 \\
2\end{array}$ & 3.88 & $3.6 \times 10^{-5}$ & 16.9 & 3.68 & $7.5 \times 10^{-5}$ & 1556 \\
\hline 8 & $\begin{array}{c}16.7 \\
5\end{array}$ & 3.88 & $3.6 \times 10^{-5}$ & 16.8 & 3.77 & $\begin{array}{c}5.47 \times 10^{-} \\
5\end{array}$ & 1558 \\
\hline 10 & $\begin{array}{c}16.7 \\
9\end{array}$ & 3.88 & $\underset{5}{3.66 \times 10^{-}}$ & 16.6 & 3.74 & $\underset{5}{6.05 \times 10^{-}}$ & 1560 \\
\hline
\end{tabular}

Table 2 Performance of down wavelength conversion, at input power and wavelength of $0 \mathrm{dBm}, 1555 \mathrm{~nm}$ respectively and pump power is $-15 \mathrm{dBm}$, at different wavelength shift "detuning".

\begin{tabular}{|c|c|c|c|c|c|c|c|}
\hline \multirow{3}{*}{$\begin{array}{c}\text { shifted } \\
\text { wavelen } \\
\text { gh } \mathrm{nm}\end{array}$} & \multicolumn{7}{|c|}{ Down wavelength conversion } \\
\hline & \multicolumn{3}{|c|}{ Forward "co-propagation" } & \multicolumn{3}{|c|}{$\begin{array}{c}\text { Back ward "counter- } \\
\text { propagation }\end{array}$} & \multirow{2}{*}{$\begin{array}{c}\text { converted } \\
\text { Waveleng } \\
\text { th } \\
\mathrm{nm}\end{array}$} \\
\hline & $\begin{array}{c}\eta \\
(\mathrm{dB})\end{array}$ & $\begin{array}{c}\text { Qualit } \\
\mathrm{y} \\
\text { factor }\end{array}$ & BER & $\begin{array}{c}\eta \\
(\mathrm{dB})\end{array}$ & $\begin{array}{c}\text { Qualit } \\
y \\
\text { factor }\end{array}$ & BER & \\
\hline 2 & 16.84 & 3.82 & $4.5 \times 10^{-5}$ & 15.6 & 3.8 & $\underset{5}{5.05 \times 10^{-}}$ & 1553 \\
\hline 4 & 16.96 & 3.89 & $3.4 \times 10^{-5}$ & 15.9 & 3.71 & $\underset{5}{7.16 \times 10^{-}}$ & 1551 \\
\hline 6 & 16.84 & 3.95 & $2.7 \times 10^{-5}$ & $\begin{array}{c}16.2 \\
2\end{array}$ & 3.9 & $3.1 \times 10^{-5}$ & 1549 \\
\hline 8 & 16.7 & 3.96 & $\underset{5}{2.67 \times 10^{-}}$ & $\begin{array}{c}16.4 \\
2\end{array}$ & 4.04 & $1.9 \times 10^{-5}$ & 1547 \\
\hline 10 & 16.5 & 4 & $2.3 \times 10^{-5}$ & $\begin{array}{c}16.5 \\
1\end{array}$ & 3.88 & $3.6 \times 10^{-5}$ & 1545 \\
\hline
\end{tabular}

Moreover it found that the area of gain variation is the best area to choose the pump power to get maximum conversion efficiency, maximum Quality factor and consequently minimum bit error rate. The area of gain variation is limited between pump power values from -20 $\mathrm{dBm}$ to $-10 \mathrm{dBm}$. Special investigation is done at the pump power $-15 \mathrm{dBm}$ at a value intermediate between -20 $\mathrm{dBm}$ and $-10 \mathrm{dBm}$. All simulations in this study are performed by optisystem ver. 7 .

\section{References}

[1] J. M. Wiessenfeld and B. Glance "Cascadability and Fanout of Semiconductor Optical Amplifier Wavelength Shifter," IEEE Photonics Technology Letters, vol. 4, no. 10, pp. 1168-1171, Oct.1992.

[2] B. Ramamurthy and B. Mukherjee" Wavelength Conversion in WDM Networking" IEEE Journal on Selected Areas in Communications, vol. 16, no. 7, pp.1060-1073, Sep.1998.
[3] A. Hamie, A. Sharaiha, M. Guegan, and J. Le Bihan "All-Optical Inverted and No inverted Wavelength Conversion using TwoCascaded Semiconductor Optical Amplifiers" IEEE Photonics Technology Letters, vol.17, no.6, pp. 1229-1231, June 2005.

[4]F. D. Mahad, A. S. M. Supa, at, S. M. Idrus, D.Forsyth "Comparative Performance Testing of SOA Wavelength Conversion Techniques for Future all-Optical Systems" Optik, vol. 124, pp. 1254-1259, 2013.

[5] A. Rani, Mr. S. Dewra" Semiconductor Optical Amplifiers in Optical Communication system-Review" International Journal of Engineering Research \& Technology (IJERT), vol. 2 no 10, pp. 2710-2719, Oct. 2013.

[6] A. Kaur "Modeling of Semiconductor Optical Amplifier Gain Characteristics for Amplification and Switching" International journal of Advanced Research in computer Science and software Engineering, vol. 6, no.4, pp. 255257, Apr. 2016.

[7] V. Thakur, G. Pandove and T. Gupta"Optical wave length converters Based on Cross Gain modulation and Cross Phase modulation in SOA" International Journal of electronics and computer sience Engineering, vol.1, no.3, pp. 1274-1280, ISSN-2277-1956. 
[8] K. Zhang, J. shao"Research on a scheme of Generating ultrawide band doublet signal based on the Cross-Gain modulation In semiconductor optical amplifier" Optical Applicata, vOl. XLVIII, no. 1, pp. 127-135, 2018.

[9] Y. said, H. Rezig and A.Bouallegue"Performance Evaluation of wavelength conversion using wide band semi conductor optial amplifier at $40 \mathrm{Gbit} / \mathrm{s}$ " the open optics Journal, vol. 4, pp. 21-28, 2010.

[10] V. Krishna, Pournami s and Resmy "FWM suppression on wavelength converters using modulation techniques" IOSR Journal of electronics and communication Engineering (IOSR-JECE), e-ISSN:2278-2834， P-ISSN:2278-8735, pp.85-89, 2017. 